\title{
Impact of patient programs on adherence and persistence in inflammatory and immunologic diseases: a meta-analysis
}

This article was published in the following Dove Press journal:

Patient Preference and Adherence

II March 2015

Number of times this article has been viewed

\author{
Chakkarin Burudpakdee ${ }^{1,2}$ \\ Zeba M Khan ${ }^{3}$ \\ Smeet Gala' \\ Merena Nanavaty' \\ Satyin Kaura ${ }^{3}$
}

'Market Access Solutions, LLC, Raritan, NJ, USA; ' ${ }^{2}$ niversity of North Carolina at Charlotte, Charlotte, NC, USA ${ }^{3}$ Celgene Corporation, Summit, NJ, USA
Correspondence: Chakkarin

Burudpakdee

IMS Health, 8280 Willow Oaks

Corporate Drive, STE 775 Fairfax, VA

2203I, USA

Tel +l 7039921028

Email cburudpakdee@us.imshealth.com
Objectives: Patient adherence and persistence is important to improve outcomes in chronic conditions, including inflammatory and immunologic (I\&I) diseases. Patient programs that aim at improving medication adherence or persistence play an essential role in optimizing care. This meta-analysis assessed the effectiveness of patient programs in the therapeutic area of I\&I diseases.

Methods: A global systematic literature review was conducted with inclusion criteria of: patient programs in I\&I diseases; published in English language between January 2008 and September 2013; and reporting measures of adherence or persistence, including medication possession ratio $>80 \%$ and persistence rate. A meta-analysis was performed using a random effects model. Subgroup analyses based on the type of program was performed whenever feasible.

Results: Of 67 studies reviewed for eligibility, a total of 17 studies qualified for inclusion in the meta-analysis. Overall, patient programs increased adherence (odds ratio $[\mathrm{OR}]=2.48,95 \%$ confidence interval $[\mathrm{CI}]=1.68-3.64, P<0.00001$ ) as compared with standard of care. Combination patient programs that used both informational and behavioral strategies were superior in improving adherence $(\mathrm{OR}=3.68,95 \% \mathrm{CI}=2.20-6.16, P<0.00001)$ compared with programs that used only informational $(\mathrm{OR}=2.16,95 \% \mathrm{CI}=1.36-3.44, P=0.001)$ or only behavioral approaches $(\mathrm{OR}=1.85,95 \% \mathrm{CI}=1.00-3.45, P=0.05)$. Additionally, patients were more likely to be persistent $(\mathrm{OR}=2.26,95 \% \mathrm{CI}=1.16-4.39, P=0.02)$ in the intervention group as compared with the control group. Persistence (in days) was significantly $(P=0.007)$ longer, by 42 additional days, in the intervention group than in the control group.

Conclusions: Patient programs can significantly improve adherence as well as persistence in the therapeutic area of I\&I diseases. Programs employing a multimodal approach are more effective in improving adherence than programs with informational or behavioral strategies alone. This in turn may improve patient outcomes.

Keywords: systematic literature review, informational, behavioral, patient interventions

\section{Introduction}

Patient adherence and persistence to treatment are important for effective disease management, especially in chronic diseases that may become more severe over time, such as autoimmune and inflammatory conditions. Adherence refers to the act of conforming to recommendations made by the provider with respect to timing, dose, and frequency of administration. ${ }^{1}$ Persistence is defined as the duration of time from initiation to discontinuation of therapy. ${ }^{1}$ Significant evidence suggests that nonadherence is highly prevalent in medical care ${ }^{2}$ and is a rising concern to health care providers and payers because it increases the cost of care and results in poor patient outcomes. ${ }^{3}$ Patients are nonadherent to treatment due to various self-identified reasons, 
including fear of side effects; poor memory; inability to pay for medications; concerns about medications, due to little or no education regarding the disease or regimen; and lack of perceived need. ${ }^{4}$

Inflammatory and immunologic (I\&I) diseases share common characteristics in that these disorders are caused by an immune system attack on the body's own tissues, leading to increased inflammation. Prevalence rates of autoimmune diseases range from five to 500 per $100,000 .{ }^{5}$ Inflammatory diseases are a significant clinical burden due to the high prevalence and incidence rates and the chronic nature of these conditions. In the USA, over seven million individuals suffer from inflammatory rheumatic diseases, which are the most severe among the inflammatory diseases. ${ }^{6}$

Adherence and persistence to long-term treatment are commonly required for optimal disease management. However, patient adherence has been shown to be suboptimal in I\&I diseases, such as psoriatic and rheumatic diseases, multiple sclerosis (MS), osteoporosis, and inflammatory bowel disease..$^{7-10}$ Only about $50 \%$ of patients adhere to prescribed medications, while $30 \%$ of patients with I\&I chronic diseases miss at least one dose intentionally or unintentionally. ${ }^{11}$ Nonadherence rates range from $43 \%$ to $72 \%$ in inflammatory bowel disease, ${ }^{12}$ from $14 \%$ to $67 \%$ in psoriasis, ${ }^{13}$ and from $30 \%$ to $80 \%$ in rheumatoid arthritis (RA). ${ }^{14,15}$ These rates highlight the need to improve adherence in patients who require long-term treatment.

A number of simple and complex programs have been developed to improve medication adherence and persistence, focusing on informational, behavioral, and combined strategies. ${ }^{16}$ Informational programs focus on increasing patient knowledge of their disease, treatments, and management tools, through educational brochures, group-based discussion sessions, ${ }^{17}$ and web-based presentations. ${ }^{9,18}$ Behavioral programs involve individually tailored adherence-focused sessions and nurse-assisted patient support programs. ${ }^{10,19}$

The effectiveness of these programs in improving adherence and persistence has not been previously assessed using a systematic approach. Therefore, a meta-analysis was undertaken to quantify and compare the impact of informational, behavioral, and combined patient programs on adherence and persistence.

\section{Methods}

\section{Search strategy}

Studies published between January 2008 and September 2013 were identified using the PubMed database. Key search terms and Medical Subject Headings (MeSH) terms (Table S1) for I\&I (eg, "ankylosing spondylitis", "psoriasis", "psoriatic arthritis", and "rheumatoid arthritis") and type of adherence and persistence program interventions (eg, "compliance", "medication adherence", "behavioral intervention", and "persistence") were used to identify relevant studies. Additionally, an internet search targeting adherence and persistence programs was conducted, and conference proceedings in the I\&I therapeutic area were searched.

Inclusion criteria included: (1) studies focused on diseases in the I\&I therapeutic area; (2) studies reporting adherence or persistence outcomes; (3) patient programs or interventions comparing exposure with a control group; and (4) studies published in the English language. Studies were not limited to any age range. Two researchers screened the titles and abstracts to determine eligibility for full text review; any disagreements were resolved by consensus with a third researcher. All studies that met our inclusion criteria were reviewed via full-text screening.

\section{Data abstraction}

Data from selected studies were abstracted, and information was collected on the country of investigation, disease, study design, sample size in the intervention and control arms, program strategy in brief, study follow-up duration, adherence and persistence definitions, and adherence and persistence results.

\section{Statistical analysis}

Statistical analysis was performed using Review Manager 5.2. Cochran Q $\chi^{2}$ and $I^{2}$ statistics were used to assess the heterogeneity among studies. Since included studies varied in the diseases studied, interventions utilized, study population, and other observable and unobservable factors, a random effects model was employed to allow study outcomes to vary assuming a normal distribution among study populations. If adherence or persistence was reported as a binary measure, then the effect of the intervention was measured as an odds ratio (OR); if it was reported as a continuous measure, then the effect was measured as the mean difference between the intervention and the control group. The pooled effect for each grouping of trials was derived from the OR for each separate trial, weighted by the inverse of the variance ( $1 /$ standard error of the mean $[\mathrm{SE}]^{2}$ ), and $95 \%$ confidence intervals (CIs) were calculated. In studies reporting discontinuation rates, a persistence rate was calculated as 1 - discontinuation rate. ${ }^{20}$

Subgroup analyses were performed based on the type of strategy implemented in the patient programs (informational, behavioral, or combined). Informational interventions comprised educational materials administered via various 
means and formats: oral and telephone communication, written materials, audiovisual presentations, and mailed or emailed materials. Behavioral interventions comprised dosing change, dosing recommendations, and treatment reminders given by telephone or email. ${ }^{21,22}$

Publication bias was evaluated using funnel plots. The overall risk of bias for an individual study was categorized as low, unclear, or high, as per the Cochrane "Risk of bias" assessment tool in Review Manager. ${ }^{23}$

\section{Results}

\section{Study selection}

Of the 67 studies that qualified for full-text screening, 17 were included in the meta-analysis. ${ }^{7-9,17-19,24-34}$ A flow diagram summarizing the study selection and inclusion is reported in Figure 1.

\section{Study characteristics}

Table 1 lists the characteristics of the 17 studies selected. Among these studies, eight were conducted in an osteoporosis patient population, ${ }^{9,17,24-26,31,33}$ four in ulcerative colitis (UC), ${ }^{18,19,29,30}$ two in MS, ${ }^{8,34}$ and one each in RA, ${ }^{32}$ childhood-onset systemic lupus erythematosus, ${ }^{28}$ and RA/ psoriasis. ${ }^{7}$ Of these 17 studies, eight used informational interventions, ${ }^{7,9,17-19,24-26}$ two used behavioral strategies, ${ }^{27,28}$ and seven used combined strategies..$^{8,29-34}$ Nine studies measured only adherence,,$^{7,17-19,25,26,28-30}$ while four studies measured only persistence, ${ }^{8,32-34}$ and four studies measured both adherence and persistence..$^{9,24,27,31}$
Table 2 reports the adherence and persistence data, including their definitions, in the studies. The follow-up period in these studies ranged from 3 to 24 months. The majority of studies used a consistent approach to measure adherence or persistence within the study, except for two studies. Among these two studies, one study by Homer et $\mathrm{al}^{7}$ measured adherence in several ways: patients were asked to record how they took their medications in a dairy; the diaries were checked against pill counts at scheduled monthly follow-up appointments, and pills were counted by investigators. Patients who altered or stopped their medication as a result of contacting the Help line or of medical advice from their general practitioner were considered to be adherent. Another study by Lai et $\mathrm{al}^{24}$ measured adherence by three methods: direct reporting (asking participants how many doses they had missed); pill counts; and self-recording (participants recorded the date on which they took their dose). From both of these studies, only the adherence measures related to pill counts performed by the investigator was used in the meta-analysis.

\section{Effect of patient programs on adherence measure}

Adherence data (\% adherent) were obtained from 13 studies, which were grouped by type of program: informational $(n=8)$; behavioral $(n=2)$; and combined $(n=3)$. Forest plots (Figure 2$)$ were developed in order to compare the ORs from each study. Overall, patient programs increased adherence $(\mathrm{OR}=2.48$, 95\% CI=1.68-3.64, $P<0.00001)$ when compared with the control group (standard prescribed care or no intervention).
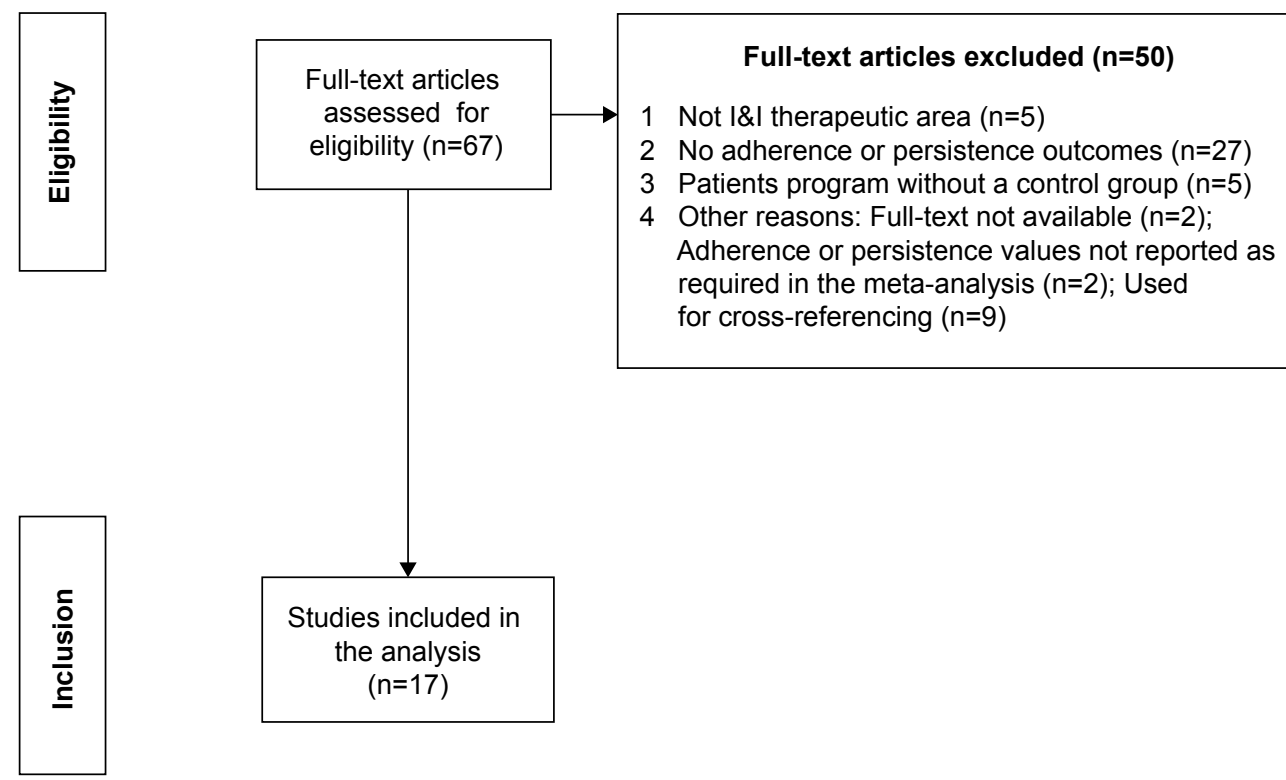

Figure I Flow diagram of systematic literature search. Abbreviation: I\&I, inflammatory and immunologic. 


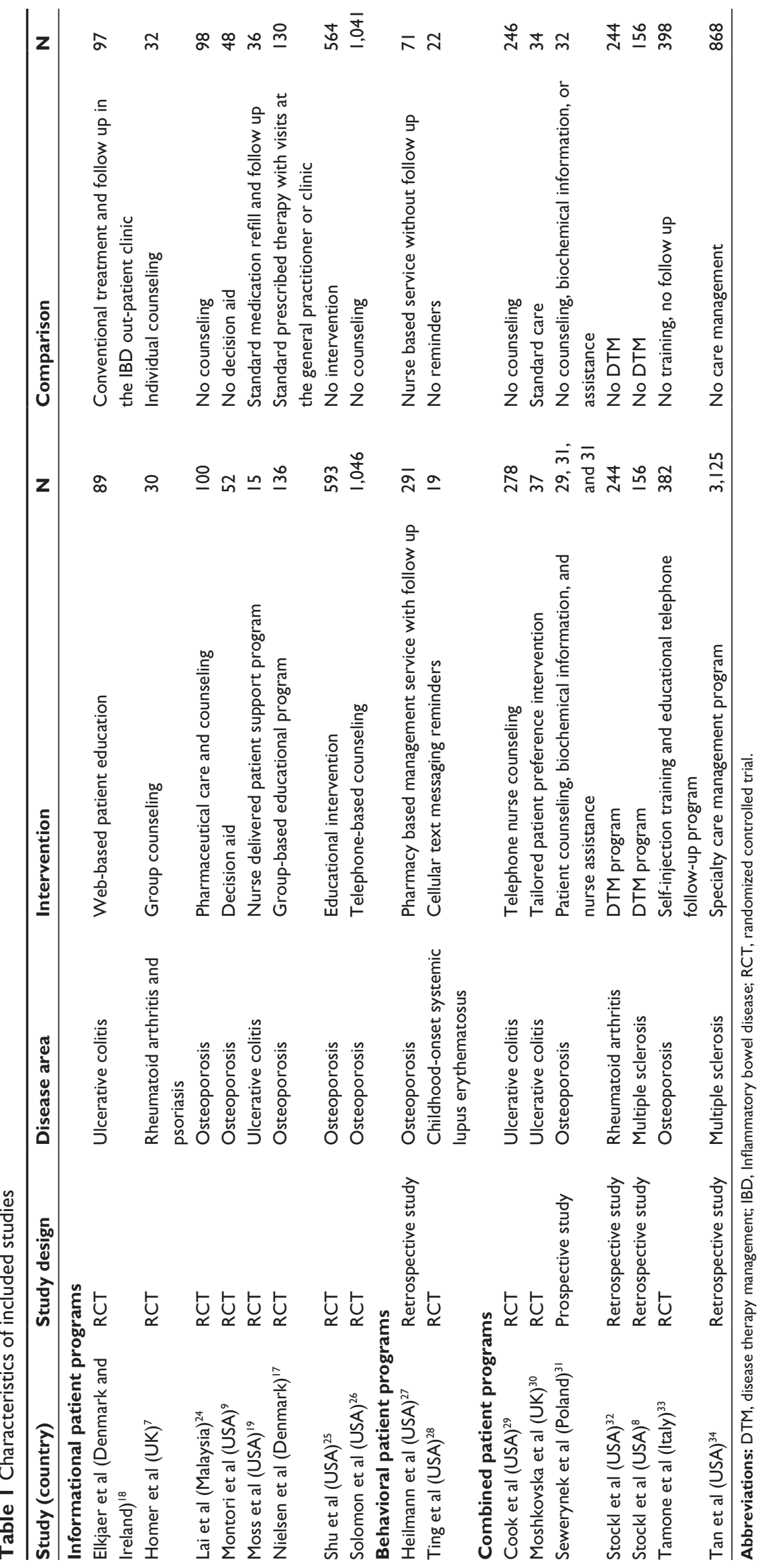




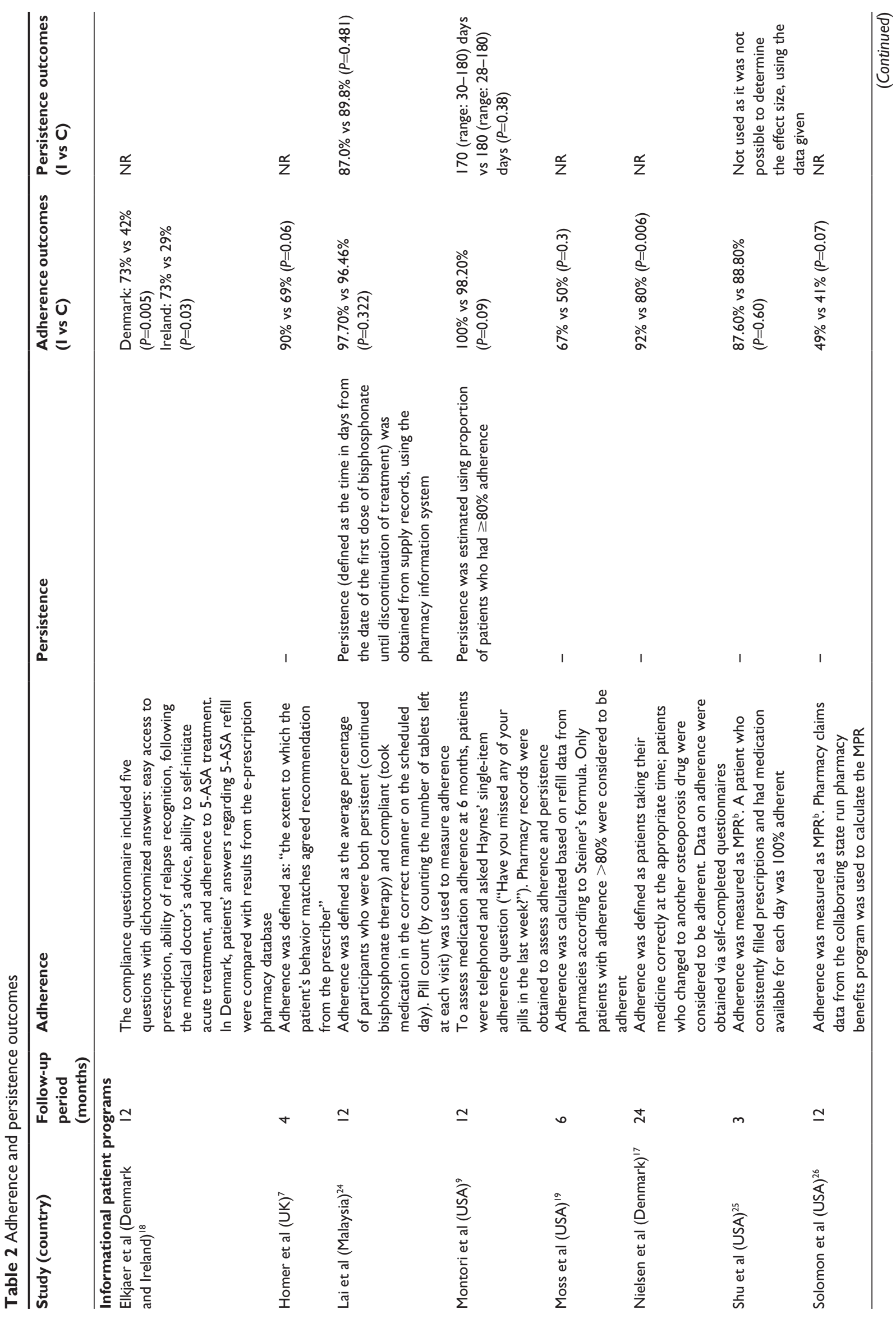




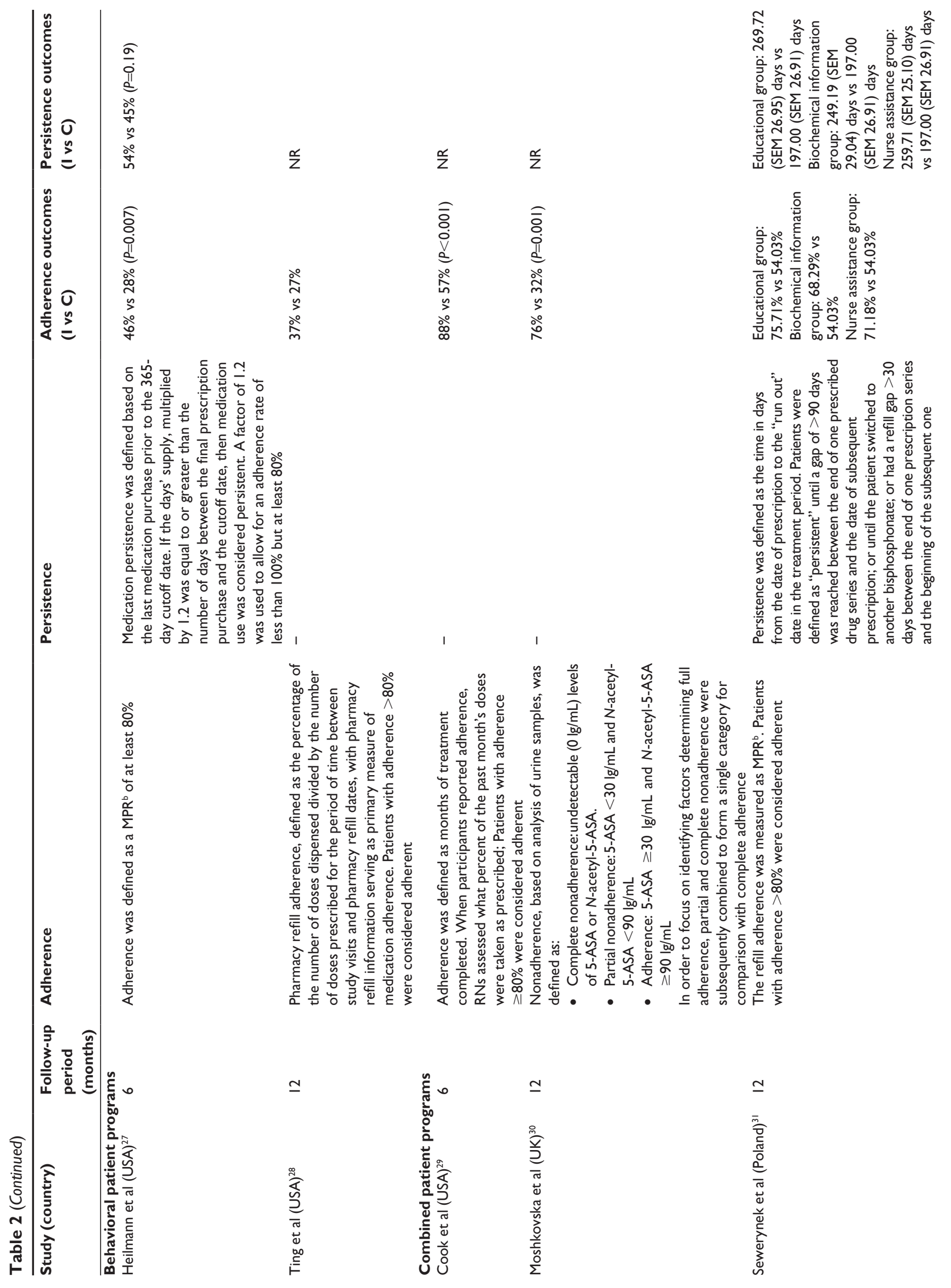




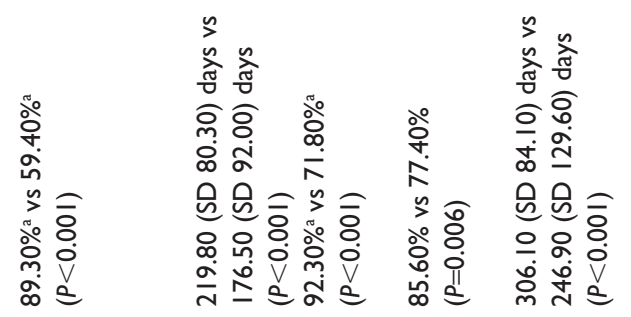

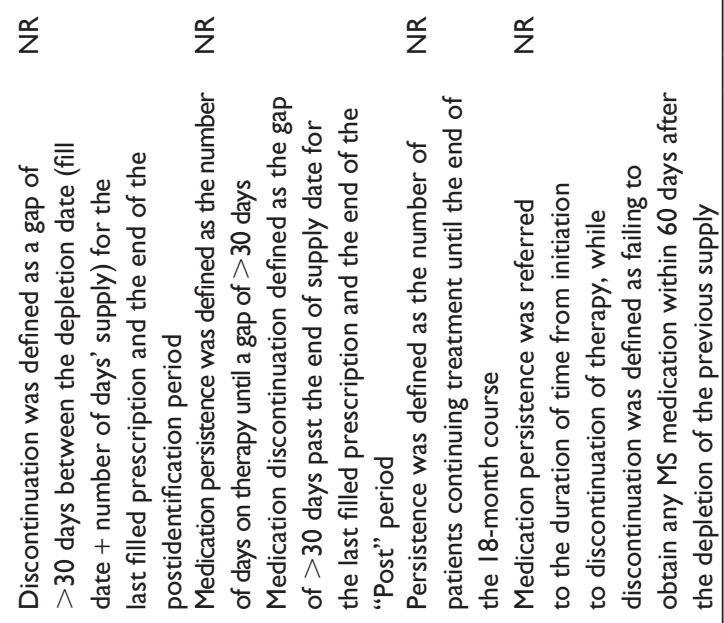

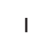

$\infty$

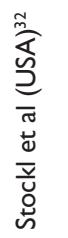

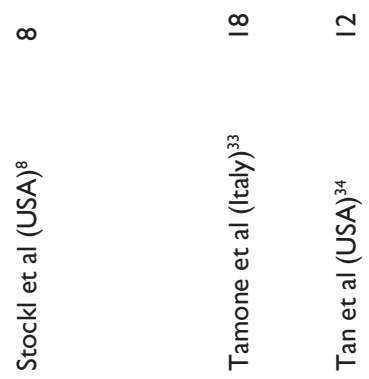

Combination patient programs that used both informational and behavioral strategies were superior in improving adherence $(\mathrm{OR}=3.68,95 \% \mathrm{CI}=2.20-6.16, P<0.00001)$ compared with programs using only informational approaches (OR=2.16, 95\% $\mathrm{CI}=1.36-3.44, P=0.001)$ or only behavioral approaches $(\mathrm{OR}=1.85,95 \% \mathrm{CI}=1.00-3.45, P=0.05)$. A random effects model was used due to the high level of heterogeneity in the overall analysis $\left(I^{2}=80 \%\right)$.

\section{Informational patient programs}

A total of seven out of eight studies reported higher adherence in the intervention group compared with the control group (Figure 2). Elkjaer et al ${ }^{18}$ employed a web-based patient education program to educate UC patients about their disease. Training was provided, and patients could ask their web doctor questions via email or text. During the training, investigators aimed to ensure that each patient understood the web-based training and education, could recognize a relapse, and was able to start the program-recommended treatment. Homer et $\mathrm{al}^{7}$ used group counseling to improve adherence in patients with RA or psoriasis. Patients were allowed to bring a relative, caregiver, or friend to these sessions. They were shown a presentation of "frequently asked questions", and individualized advice was provided to each. Lai et $\mathrm{a}^{24}$ distributed a "counseling package" to the participants, which consisted of an explanation of osteoporosis, risk factors, lifestyle modifications, goals of osteoporosis therapy, side effects, and the importance of medication adherence. Verbal counseling was reinforced with an osteoporosis booklet, and pharmacists followed up with participants. Montori et $\mathrm{al}^{9}$ used a decision aid, which was a tailored pictographic that illustrated their 10-year fracture risk estimate, absolute risk reduction with bisphosphonates, side effects, and outof-pocket costs. The decision aid also showed the absolute risk reduction in fracture risk with alendronate, assuming a treatment-related reduction in overall osteoporotic fracture risk of $40 \%$. Moss et $\mathrm{a}^{19}$ assessed patients enrolled in the Script Assist program (an independent treatment adherence program that provides disease-specific information and promotion of medication adherence to patients). Patients received phone calls at 24 hours, 3 weeks, 7 weeks, 15 weeks, and 23 weeks after enrollment, from nurses who were trained to assess patient risk for noncompliance and to intervene with psychological techniques that could improve medication persistence. Nielsen et a $1^{17}$ conducted a group-based educational program in classes of eight to 12 patients each, lasting 3 to 4 hours a week over 4 weeks, conducted by a multidisciplinary team of physicians, dieticians, physiotherapists, and 


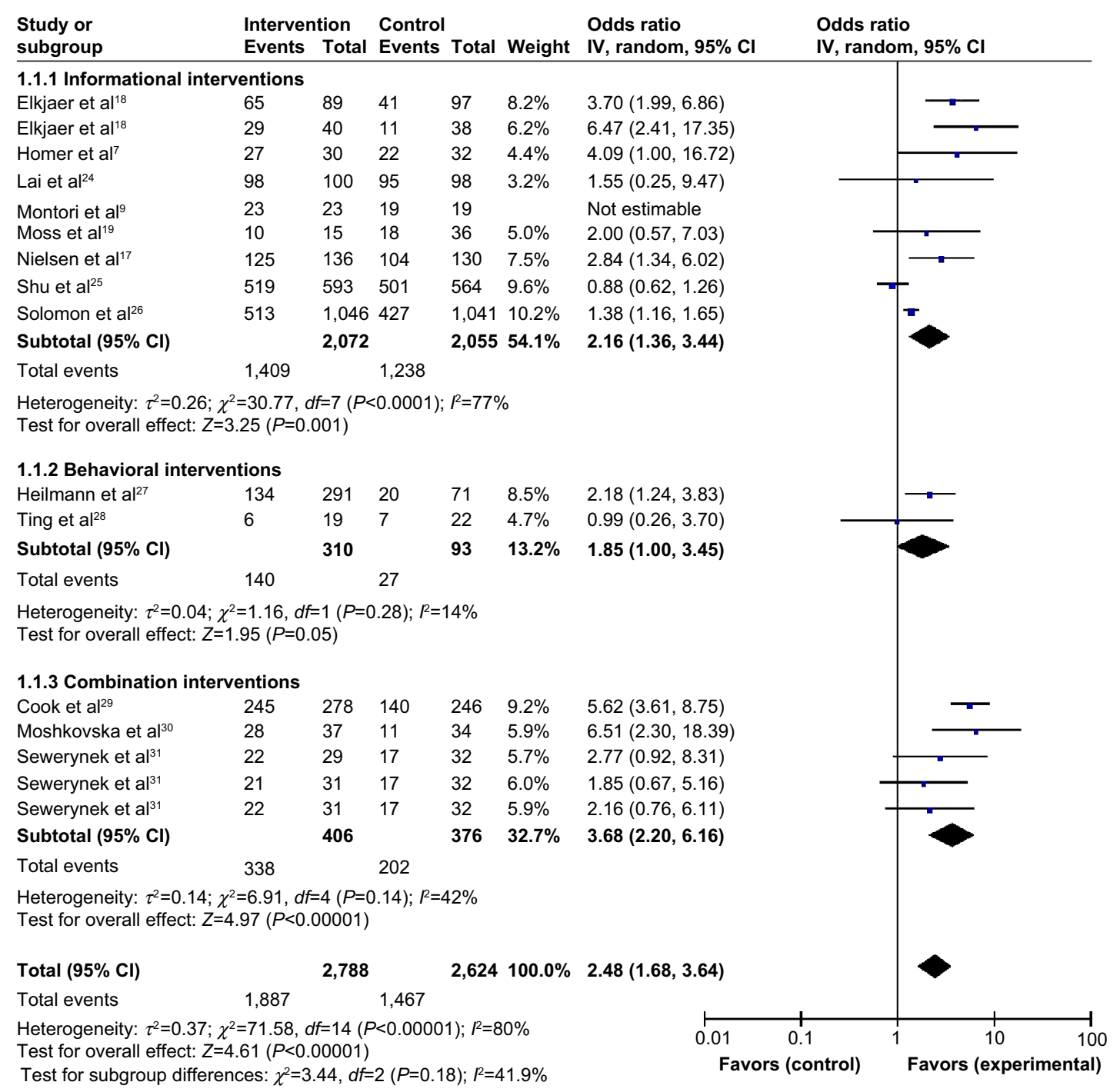

Figure 2 Program effectiveness on adherence, by type of patient program.

Notes: The study by Sewerynek et $\mathrm{al}^{32}$ is one study with three intervention groups: (1) the patient counseling group; (2) the biochemical information groups; and (3) the nurse-assistance group. The squares in the lines represent the effect estimate, the lines represent the length of the confidence interval, the diamonds represents the overall result of the meta-analysis.

Abbreviations: $\mathrm{Cl}$, confidence interval; IV, inverse variance.

nurses, which were adjusted according to individual patient backgrounds and needs in order to strengthen competence and empowerment. Additionally, patients were invited to participate in a computerized support program, where patients were contacted once a month for 4 months and asked about pain, quality of life, and physical activity. Solomon et $\mathrm{al}^{26}$ sent out seven informational mailings regarding topics such as exercise, fall prevention, and recommended calcium intake to all the study patients. Additionally, the intervention group received ten motivational interview counseling sessions via telephone with a health educator, where each session had a specific educational topic (discussing medications with physician, calcium and vitamin D supplementation, fall prevention, managing adverse effects of medication, etc) and included a series of open-ended questions to elicit subjects' attitudes toward medication adherence and to determine barriers to long-term osteoporosis medication use.

Shu et $\mathrm{a}^{25}$ reported lower adherence in the intervention group compared with the control group. In this study, randomly selected primary care physicians and their patients received education about osteoporosis diagnosis and treatment. The primary care physicians also received face-to-face education by trained pharmacists, while patients received letters outlining the importance of osteoporosis, its diagnosis, and appropriate treatment, and automated calls inviting them to undergo bone mineral density testing. The pharmacists had participated in a 1-day training session and several follow-up teleconferences about osteoporosis and the principles of one-to-one physician education. The control group received no education. 


\section{Behavioral patient programs}

Heilmann et $\mathrm{a}^{27}$ used a pharmacy-based management service, where a clinical pharmacist developed a therapeutic plan for treatment recommendations (bone marrow density screening, initiation of osteoporosis therapy, and calcium and vitamin D supplementation) after reviewing the medical history of patients. This plan was then approved by the primary care provider before implementation with patients. Ting et $\mathrm{al}^{28}$ sent daily text messages to patients three times a week prior to each scheduled follow-up clinic appointment. These texts were individualized for each patient by including the scheduled time of the upcoming clinic appointment. Messages were also sent in cases when patients failed to schedule a follow-up visit. A standardized daily reminder was sent to patients based on the prescription (eg once or twice per day), and also received printed information about the benefits and the side effects of the medication.

\section{Combined patient programs}

Studies conducted by Sewerynek et al, ${ }^{31}$ Cook et al, ${ }^{29}$ and Moshkovska et al ${ }^{30}$ evaluated the impact of combined strategies on patient adherence. In the study conducted by Cook et al ${ }^{29}$ telephone follow-up calls were made to UC patients. Within a day of referral, patients received a call from a trained registered nurse who provided an introduction to the program, a preliminary assessment, and offer of a patient program, based on their concerns and readiness for change, using cognitive-behavioral and motivational interviewing counseling techniques. All patients were given a toll-free number for questions, and the referring health care practitioner received a progress note after each call, with notes on the participant's adherence level and concerns. Moshkovska et a ${ }^{30}$ employed a tailored patient preference program in which UC patients were given one-on-one education and motivational sessions to deliver individualized support, motivation, and education. At week 4, a brief follow-up telephone call was made to the patient, and at week 24, a 10-minute reinforcement session was held to stress the importance of adherence to medication, to reassess beliefs regarding medicine-taking, and to discuss practical problems. At the end of the session, patients were offered an educational leaflet and a choice of three practical adherence-enhancing patient programs that included medication reminder charts, visual medication reminders for refrigerators and bedside cabinets, daily or weekly electronic pill box organizers with alarms, and a mobile telephone alarm setup.

Sewerynek et $\mathrm{al}^{31}$ randomized patients into four groups, receiving: patient counseling; biochemical information; nurse assistance; or no intervention (control). In the nurse assisted group, a follow-up phone contact was made after 3 and 9 months of treatment, to improve monitoring. In the counseled group, patients were educated and interviewed for 30 minutes about osteoporosis, diagnostic methods, treatment, and preventative behavior. In the biochemical group, patients were educated about serum levels of calcium, phosphorus, alkaline phosphatase, and of urinary calcium and phosphorus concentration levels and diurnal excretion rates.

\section{Effect of patient programs on persistence}

Persistence in studies was measured as either percent of patients who were persistent or number of days persistent; the former was a dichotomous variable, while the latter was a continuous variable. As shown in Figure 3, patients were more likely to be persistent $(\mathrm{OR}=2.26,95 \% \mathrm{CI}=1.16-4.39, P=0.02)$ in the intervention group compared with the control group. A subgroup analysis was not feasible due to the small number of studies reporting persistence. A random effects model was used to adjust for the presence of heterogeneity $\left(I^{2}=86 \%\right)$.

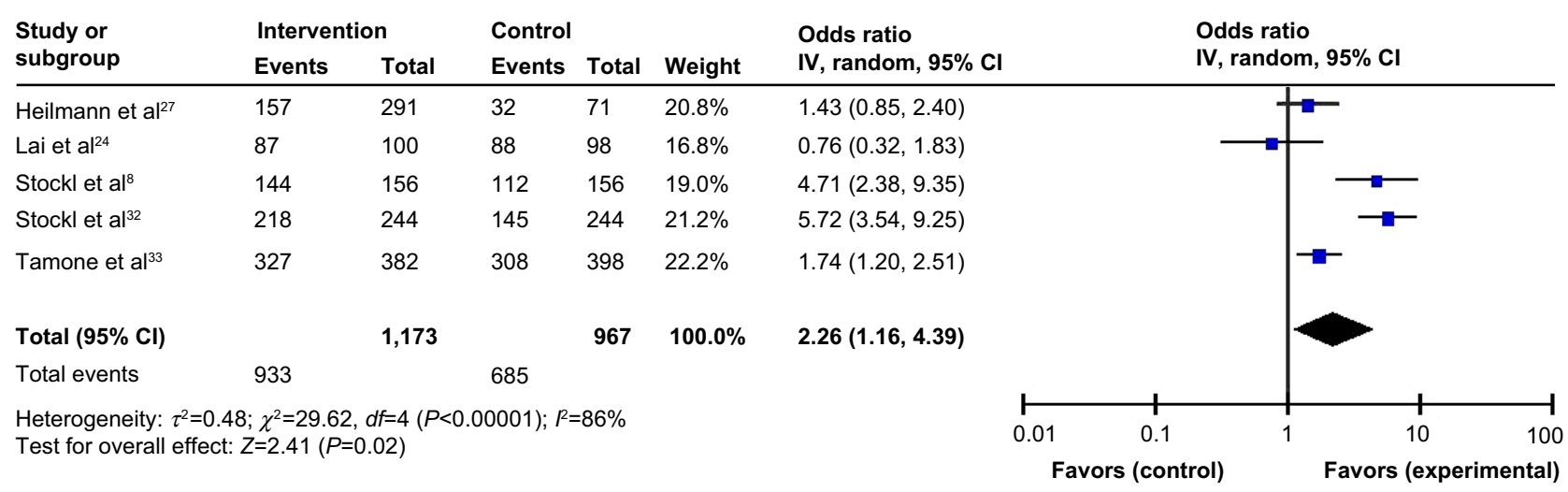

Figure 3 Program effectiveness measured as \% persistence.

Notes: The squares in the lines represent the effect estimate, the lines represent the length of the confidence interval, the diamonds represent the overall result of the meta-analysis.

Abbreviations: $\mathrm{Cl}$, confidence interval; IV, inverse variance. 
Five studies measured persistence in terms of percent of patients persistent over follow up. Stockl et $\mathrm{al}^{8}$ studied MS patients in a disease therapy management (DTM) program, who received telephone consultations, mailed care plans, and educational materials, based on a predefined schedule for level of intensity of the program (regular-intensity versus highintensity). In another study by Stockl et al patients with RA were enrolled in a DTM program in which they were given a brochure detailing medication ordering and storage, monitoring, proper disposal of ancillary supplies, mail service medication delivery, refill reminders by patient care coordinators, and access to a pharmacist 24 hours a day, 7 days a week. ${ }^{32}$ The program used a patient-centric approach providing education and support, to assist patients in developing self-management skills for symptom and treatment management. Each patient was assigned a clinician for the entirety of the program and received telephone consultations (licensed pharmacist or registered nurse) providing education on the medical condition and treatment options, and promoting medication adherence. Tamone et $\mathrm{al}^{33}$ implemented an educational telephone program. At the beginning of treatment, nurses trained patients on selfinjection, then, every 2 months, nurses gave new drug pens to the patients; this guaranteed the surveillance of compliance. Nurses called patients to help resolve any issues, schedule the next visit, and, if applicable, collect adverse events information, dates, and reasons for treatment discontinuation.

In a small number of studies, persistence was also reported as number of days persistent (Figure 4). When measured as persistent days, persistence was significantly longer, by 41.96 additional days $(P=0.007)$, in the intervention group than in the control group.

As described above, Montori et $\mathrm{al}^{9}$ employed a pictographic decision aid; Sewerynek et $\mathrm{al}^{31}$ employed three intervention groups in which patients received either nurse assistance, information about their biochemical charts, or counseling; and the study by Stockl et $\mathrm{al}^{8}$ of (MS), utilized a DTM program. Tan et $\mathrm{al}^{34}$ provided a specialty care management program service, including mail order medications, disease-specific patient education materials, refill reminder calls, and assessment calls by nurses at the beginning of the program and at months 3,6 , and 12 , and every 12 months thereafter.

\section{Risk of bias in the reviewed studies}

The common types of biases evaluated in this analysis were selection bias, performance bias, detection bias, attrition bias, reporting bias, and other biases inherent in interventional study designs. Selection bias was determined if patients were not assigned to an intervention or control group using random sequence generation and if the allocation of participants were not concealed. Performance bias referred to the lack of blinding of participants and personnel blinding ensures that the control group receives similar attention, treatment, and diagnostic investigations as the intervention group. Detection bias referred to blinding of investigators, which reduces confounding related to the knowledge of intervention assignment. Reporting bias is often related to selective reporting of study measures (publications more often report statistically significant differences than nonsignificant differences, leading to reporting bias), while attrition bias is due to incomplete outcomes data, due to omission of some participants from the reports of analyses. Other biases included bias due to study designs, and inclusion and exclusion of patients.

As depicted in Figure 5, $\operatorname{six}^{8,27,29,32-34}$ out of the 17 studies did not perform random sequence generation, and eleven ${ }^{8,17,19,24-28,32-34}$ did not conceal the allocation of participants, resulting in selection bias. Although blinding of participants and personnel, as well as that of outcomes

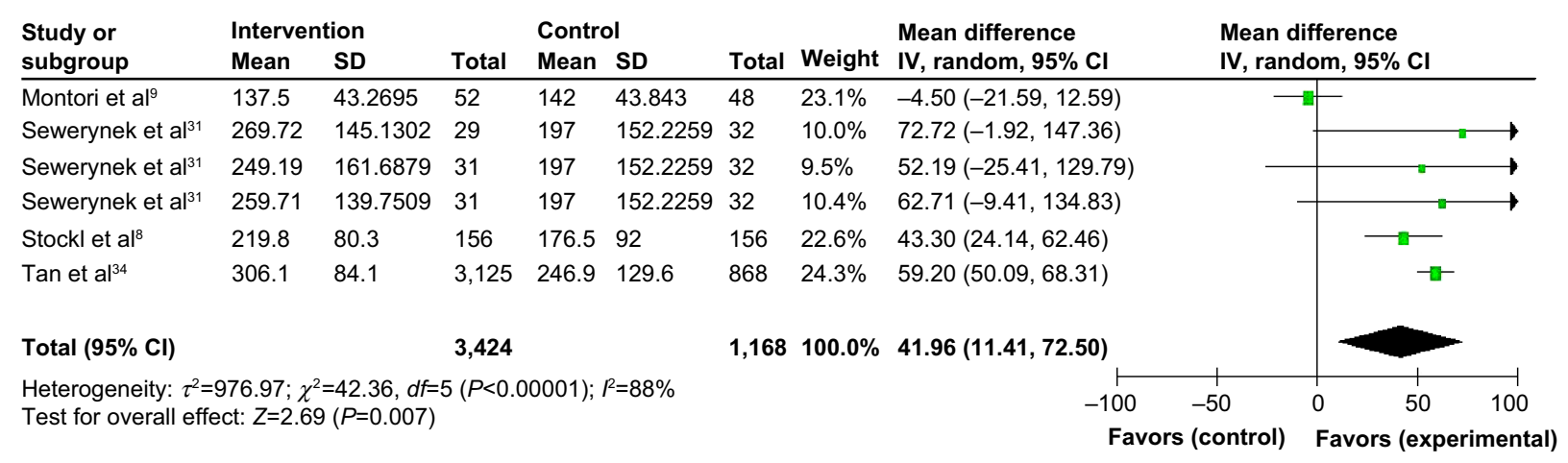

Figure 4 Effect of intervention vs control on persistence (in days) in I\&I.

Notes: The squares in the lines represent the effect estimate, the lines represent the length of the confidence interval, the diamonds represent the overall result of the meta-analysis.

Abbreviations: $\mathrm{Cl}$, confidence interval; I\&I, inflammatory and immunologic; SD, standard deviation; IV, inverse variance. 


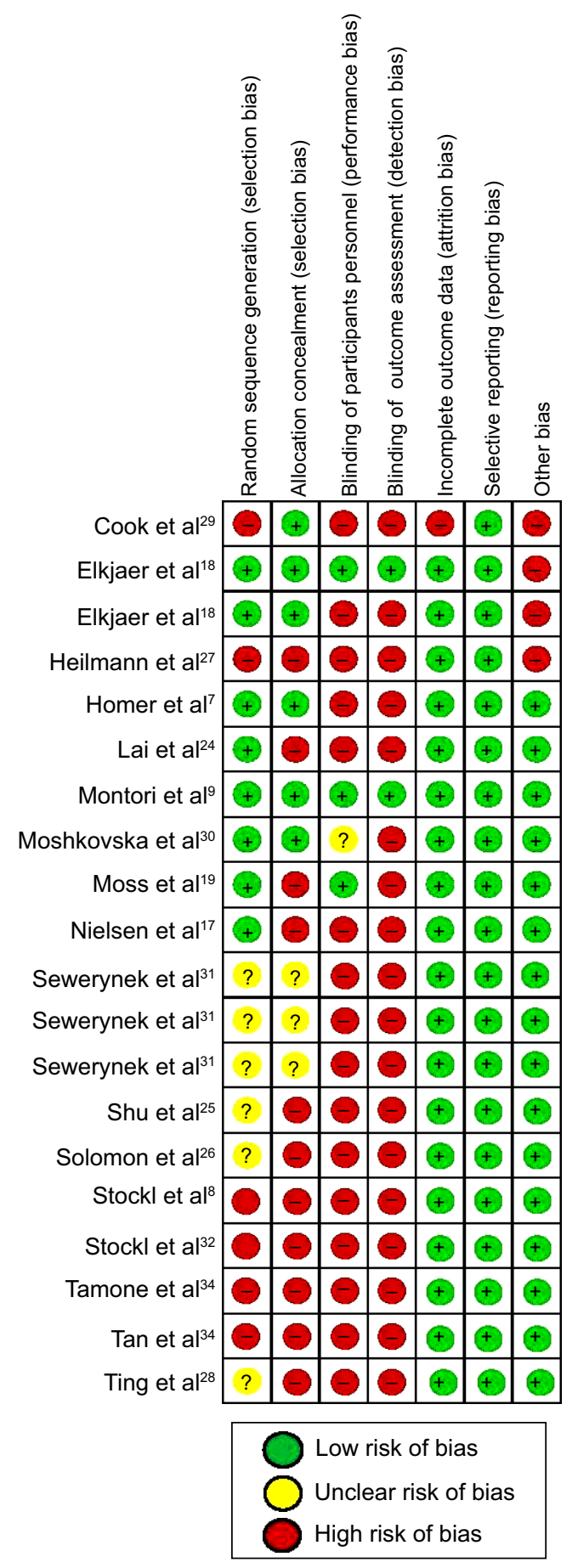

Figure 5 Risk of bias summary.

assessed in the study, is not usually possible in patient programs, it was addressed by two studies. ${ }^{9} 18$ There was no attrition or reporting bias in the selected studies. However, other types of biases related to study designs were observed in three studies.

Among these studies, Cook et al ${ }^{29}$ divided the intervention population into two groups: with high risk and low risk. However, the results were aggregated for the two groups and were not presented separately for the high- and low-risk patients. The study by Elkjaer et $\mathrm{al}^{18}$ only selected patients who suffered from mild to moderate UC; those with severe UC were not included, and yet, they are an important target population for adherence. Participants in the study conducted by Heilmann et $\mathrm{al}^{27}$ required continuous enrollment in their health plan (no membership gaps greater than 45 days), therefore, members who did not have continuous membership were not included in the study.

\section{Discussion}

\section{Key findings}

Though various patient programs have been developed with the goal of improving adherence and persistence, little has been published on the overall effectiveness of these programs in the therapeutic area of I\&I diseases. The results of this meta-analysis show that patient programs incorporating combined strategies (informational and behavioral techniques together) were more likely to improve adherence compared with informational or behavioral strategies alone.

Among the combination patient programs, two out of three studies significantly increased adherence, using cognitive-behavioral and motivational interview counseling over the telephone and tailoring the program as per patient preference to include individualized education, support sessions, and problem solving. ${ }^{29,30}$ Patient counseling and motivational sessions formed a major part of all combination patient programs, suggesting that a greater degree of communication may have existed between patients and health care practitioners in these instances. Combined strategies also focused on providing education about the disease and treatment. Therefore, behavior modification as well disease knowledge was targeted to improve adherence.

Among the eight informational patient programs, three significantly increased adherence in the intervention group as compared with the control group. These programs included strategies for web-based patient education, group counseling, and group-based education. ${ }^{7,17,18} \mathrm{We}$ found that patient education and counseling, more so in a group-based setting, can be an effective strategy to enhance adherence. One study reported lower adherence in the intervention group as compared with the control group. ${ }^{25}$ This intervention focused on physicians more than patients and suggested that adherence programs might be more effective when they are patientcentric. In the study, researchers suspected that their inability to observe a difference in adherence was due to a high rate of baseline adherence in the control group, and the 10-month follow-up period. In the study by Homer et al $^{7}$ reports by the 
participants - the use of diary and self-reports of pill countwere used, which may lead to biases. Hence, the pill count data recorded by the health care professional, considered to be unbiased, was used for the meta-analysis.

Behavioral techniques suggest a positive impact on adherence benefits when data were pooled, though our finding is based on only two studies. Of the two studies, Ting et al ${ }^{28}$ did not show a positive benefit; however, the study included a small sample size, of 19 patients in the intervention group and 22 patients in the control group. In contrast, Heilmann et $\mathrm{al}^{27}$ did show a significant improvement in adherence, and the study was weighted more heavily due to the much larger sample size ( $\mathrm{N}=362)$. The strategy included pharmacybased management services that provided recommendations on screening and medication. Also, Heilmann et a ${ }^{27}$ followed patients for 6 months, while Ting et $\mathrm{al}^{28}$ followed their patients for 12 months. The longer follow-up period may have influenced level of adherence. There was also a major difference in the patient population, with the study by Heilmann et $\mathrm{al}^{27}$ consisting of elderly and potentially more severe patients. Heilmann et $\mathrm{al}^{27}$ studied women patients suffering from osteoporosis, aged 67 years or older, while Ting et $\mathrm{al}^{28}$ included patients with childhood-onset systemic lupus erythematosus, aged between 13 and 25 years, with unlimited access to cellular text messaging.

Persistence could be enhanced significantly by DTM programs, self-injection training, disease and treatment education over the telephone, and specialty care management. DTM, as well as specialty care management programs, involve a combination of services, such as consultation, care plans, educational material mailings, reminders, mail-service medication delivery, access to the pharmacist, and thorough follow-up. ${ }^{8,32-34}$ Hence, they prove to be exhaustive programs that can improve persistence.

Overall, programs that empower patients, through counseling, education, reminders, and support, improve adherence and persistence. Additionally, active participation of pharmacists, registered nurses, and primary care physicians can further enhance adherence. Follow up is an important part of patient programs as it gives patients a chance to ask questions and resolve any issues. A systematic review conducted by Haynes et $\mathrm{a}^{21}$ confirms our findings and reported that patient programs that consisted of both informational and behavioral techniques, such as counseling, reminders, reinforcement, and individualized plans, commonly improved patient adherence. However, the review by Haynes et al ${ }^{21}$ only included randomized controlled trials and made no attempt to pool data to evaluate the effectiveness of these programs in a meta-analysis.

\section{Strengths and limitations of the study}

This meta-analysis adds to the current body of evidence by assessing the effectiveness of programs on adherence and persistence outcomes in diseases that require long-term management. One of the strengths of this study is the comprehensive, structured, and systematic approach undertaken to search the literature and conference proceedings to identify all studies that assessed adherence-focused patient programs. Furthermore, to our knowledge, this is the first meta-analysis in the therapeutic area of I\&I diseases to study the effect of such programs, based on the type of the patient program used.

Although this review provides a comprehensive understanding of the effect of patient programs on adherence and persistence, some limitations should be considered when interpreting our findings. Although osteoporosis has not been categorized as an I\&I disease, emerging molecular and clinical evidence highlights that inflammation exerts significant influence on bone turnover, which induces osteoporosis. ${ }^{35}$ Transplantation was not included in this analysis, due to the unique clinical and treatment characteristics in the transplant population, which may not be generalizable and comparable to populations with chronic I\&I disease. A limitation inherent in meta-analyses is that we could not control for potential confounding variables such as age, socioeconomic level, education level, disease severity, and comorbidities. Also, studies that reported persistence as a medication possession ratio were not included in the meta-analysis. However, excluding these studies did not likely bias our finding for an overall benefit because these studies reported a significant increase in the medication possession ratio in the intervention group compared with the control group. Additionally, a number of studies focused on a specific population segment, for example, patients with internet access or unlimited access to cellular text messaging, and results may not be generalizable to the general population. Although these patient programs were associated with improvements in adherence and persistence, it is worth noting that they were conducted in controlled settings. The actual effect of such complex patient programs on patients may be different in real-world clinical practice.

Overall, we found that patient programs can significantly improve adherence as well as persistence, in I\&I diseases. Programs employing a multimodal approach seem to be the most effective, given that they address multiple aspects of treatment management; however, informational or behavioral strategies alone also appear to be beneficial by themselves. Supporting and implementing similar patient programs may in turn improve patient outcomes in those with chronic I\&I disease. 


\section{Disclosure}

Market Access Solutions, LLC, a consulting company, received funding from Celgene Corporation to conduct the research, analysis, and develop the manuscript. Zeba M Khan and Satyin Kaura are employees of Celgene Corporation. Chakkarin Burudpakdee was formerly employed by Market Access Solutions, LLC, and is currently a Principal at IMS Health. Smeet Gala and Merena Nanavaty are employed at Market Access Solutions, LLC. The authors report no other conflicts of interest.

\section{References}

1. Cramer JA, Roy A, Burrell A, et al. Medication compliance and persistence: terminology and definitions. Value Health. 2008;11(1):44-47.

2. Fischer MA, Stedman MR, Lii J, et al. Primary medication nonadherence: analysis of 195,930 electronic prescriptions. J Gen Intern Med. 2010;25(4):284-290.

3. Ho PM, Bryson CL, Rumsfeld JS. Medication adherence: its importance in cardiovascular outcomes. Circulation. 2009;119(23):3028-3035.

4. McHorney CA, Spain CV. Frequency of and reasons for medication non-fulfillment and non-persistence among American adults with chronic disease in 2008. Health Expect. 2011;14(3):307-320.

5. Cooper GS, Stroehla BC. The epidemiology of autoimmune diseases. Autoimmun Rev. 2003;2(3):119-125.

6. Helmick CG, Felson DT, Lawrence RC, et al; National Arthritis Data Workgroup. Estimates of the prevalence of arthritis and other rheumatic conditions in the United States. Part I. Arthritis Rheum. 2008; 58(1):15-25.

7. Homer D, Nightingale P, Jobanputra P. Providing patients with information about disease-modifying anti-rheumatic drugs: Individually or in groups? A pilot randomized controlled trial comparing adherence and satisfaction. Musculoskeletal Care. 2009;7(2):78-92.

8. Stockl KM, Shin JS, Gong S, Harada AS, Solow BK, Lew HC. Improving patient self-management of multiple sclerosis through a disease therapy management program. Am J Manag Care. 2010;16(2):139-144.

9. Montori VM, Shah ND, Pencille LJ, et al. Use of a decision aid to improve treatment decisions in osteoporosis: the osteoporosis choice randomized trial. Am J Med. 2011;124(6):549-556.

10. Hommel KA, Herzer M, Ingerski LM, Hente E, Denson LA. Individually tailored treatment of medication nonadherence. J Pediatr Gastroenterol Nutr. 2011;53(4):435-439

11. Barber N, Parsons J, Clifford S, Darracott R, Horne R. Patients' problems with new medication for chronic conditions. Qual Saf Health Care. 2004;13(3):172-175.

12. Hawthorne AB, Rubin G, Ghosh S. Review article: medication nonadherence in ulcerative colitis - strategies to improve adherence with mesalazine and other maintenance therapies. Aliment Pharmacol Ther. 2008;27(12):1157-1166.

13. Thorneloe RJ, Bundy C, Griffiths CE, Ashcroft DM, Cordingley L. Adherence to medication in patients with psoriasis: a systematic literature review. Br J Dermatol. 2013;168(1):20-31.

14. van den Bemt BJ, Zwikker HE, van den Ende CH. Medication adherence in patients with rheumatoid arthritis: a critical appraisal of the existing literature. Expert Rev Clin Immunol. 2012;8(4):337-351.

15. Barton JL, Criswell LA, Kaiser R, Chen YH, Schillinger D. Systematic review and metaanalysis of patient self-report versus trained assessor joint counts in rheumatoid arthritis. J Rheumatol. 2009; 36(12):2635-2641.

16. Kripalani S, Yao X, Haynes RB. Interventions to enhance medication adherence in chronic medical conditions: a systematic review. Arch Intern Med. 2007;167(6):540-550.
17. Nielsen D, Ryg J, Nielsen W, Knold B, Nissen N, Brixen K. Patient education in groups increases knowledge of osteoporosis and adherence to treatment: a two-year randomized controlled trial. Patient Educ Couns. 2010;81(2):155-160.

18. Elkjaer M, Shuhaibar M, Burisch J, et al. E-health empowers patients with ulcerative colitis: a randomised controlled trial of the web-guided 'Constant-care' approach. Gut. 2010;59(12):1652-1661.

19. Moss AC, Chaudhary N, Tukey M, et al. Impact of a patient-support program on mesalamine adherence in patients with ulcerative colitis - a prospective study. J Crohns Colitis. 2010;4(2):171-175.

20. Kothawala P, Badamgarav E, Ryu S, Miller RM, Halbert RJ. Systematic review and meta-analysis of real-world adherence to drug therapy for osteoporosis. Mayo Clin Proc. 2007;82(12):1493-1501.

21. Haynes RB, McKibbon KA, Kanani R. Systematic review of randomised trials of interventions to assist patients to follow prescriptions for medications. Lancet. 1996;348(9024):383-386.

22. Peterson AM, Takiya L, Finley R. Meta-analysis of trials of interventions to improve medication adherence. Am J Health Syst Pharm. 2003;60(7):657-665.

23. Higgins JPT, Green S, editors. Cochrane handbook for systematic reviews of interventions. Version 5.1. 0. The Cochrane Collaboration; 2011 [updated March 2011]. Available from: www.cochrane-handbook. org. Accessed December 24, 2014.

24. Lai P, Chua SS, Chan SP. A systematic review of interventions by healthcare professionals on community-dwelling postmenopausal women with osteoporosis. Osteoporos Int. 2010;21(10):1637-1656.

25. Shu AD, Stedman MR, Polinski JM, et al. Adherence to osteoporosis medications after patient and physician brief education: post hoc analysis of a randomized controlled trial. Am J Manag Care. 2009;15(7):417-424.

26. Solomon DH, Iversen MD, Avorn J, et al. Osteoporosis telephonic intervention to improve medication regimen adherence: a large, pragmatic, randomized controlled trial. Arch Intern Med. 2012;172(6):477-483.

27. Heilmann RM, Friesleben CR, Billups SJ. Impact of a pharmacistdirected intervention in postmenopausal women after fracture. $A m J$ Health Syst Pharm. 2012;69(6):504-509.

28. Ting TV, Kudalkar D, Nelson S, et al. Usefulness of cellular text messaging for improving adherence among adolescents and young adults with systemic lupus erythematosus. J Rheumatol. 2012;39(1):174-179.

29. Cook PF, Emiliozzi S, El-Hajj D, McCabe MM. Telephone nurse counseling for medication adherence in ulcerative colitis: a preliminary study. Patient Educ Couns. 2010;81(2):182-186.

30. Moshkovska T, Stone MA, Smith RM, Bankart J, Baker R, Mayberry JF. Impact of a tailored patient preference intervention in adherence to 5-aminosalicylic acid medication in ulcerative colitis: results from an exploratory randomized controlled trial. Inflamm Bowel Dis. 2011;17(9):1874-1881.

31. Sewerynek E, Horst-Sikorska H, Stępień-Kłos W, et al. The role of counselling and other factors in compliance of postmenopausal osteoporotic patients to alendronate 70 therapy. Arch Med Sci. 2013;9(2):288-296.

32. Stockl KM, Shin JS, Lew HC, et al. Outcomes of a rheumatoid arthritis disease therapy management program focusing on medication adherence. J Manag Care Pharm. 2010;16(8):593-604.

33. Tamone C, Fonte G, Panico A, Molinatti PA, D'Amelio P, Isaia GC. Impact of a phone follow-up program on persistence with teriparatide or PTH(1-84) treatment. Calcif Tissue Int. 2012;90(4):272-278.

34. Tan H, Yu J, Tabby D, Devries A, Singer J. Clinical and economic impact of a specialty care management program among patients with multiple sclerosis: a cohort study. Mult Scler. 2010;16(8):956-963.

35. Ginaldi L, Di Benedetto MC, De Martinis M. Osteoporosis, inflammation and ageing. Immun Ageing. 2005;2(1):14. 


\section{Supplementary material}

Table SI Search terms

\begin{tabular}{ll}
\hline Primary search terms & Secondary search terms \\
\hline Allergies & Compliance \\
Ankylosing spondylitis & Medication adherence \\
Asthma & Adherence program \\
Behcet's disease & Adherence intervention \\
Bursitis & Behavioral intervention \\
Celiac disease & Capacitance \\
Chronic pain & Compliance program \\
Crohn's disease & Compliance intervention \\
Gout & Concordance \\
Idiopathic thrombocytopenic purpura & Medication possession ratio \\
Inflammatory bowel disease & Persistence \\
Multiple sclerosis & Persistence program \\
Osteoarthritis & Proportion of days covered \\
Osteoporosis & \\
Psoriasis & \\
Psoriatic arthritis & \\
Rheumatoid arthritis & \\
Sarcoidosis & \\
Scleroderma & \\
Sjögren's syndrome & \\
Systemic lupus & \\
Systemic sclerosis & \\
Tendonitis & \\
Ulcerative colitis & \\
Vasculitis &
\end{tabular}

Vasculitis

\section{Publish your work in this journal}

Patient Preference and Adherence is an international, peer-reviewed, open access journal that focuses on the growing importance of patient preference and adherence throughout the therapeutic continuum. Patient satisfaction, acceptability, quality of life, compliance, persistence and their role in developing new therapeutic modalities and compounds to optimize clinical outcomes for existing disease states are major areas of interest for the journal. This journal has been accepted for indexing on PubMed Central The manuscript management system is completely online and includes a very quick and fair peer-review system, which is all easy to use. Visit http://www. dovepress.com/testimonials.php to read real quotes from published authors.

Submit your manuscript here: http://www.dovepress.com/patient-preference-and-adherence-journal 